\title{
Incorporação de urucum como aditivo antioxidante em embalagens biodegradáveis a base de quitosana
}

\author{
The incorporation of annatto as antioxidant additive based biodegradable packaging chitosan
}

\author{
Maria Cecília Castelo Branco de Santana ${ }^{I}$ Bruna Aparecida Souza Machado II \\ Tamara do Nascimento da Silva ${ }^{\mathrm{I}}$ Itaciara Larroza Nunes ${ }^{\mathrm{II}}$ Janice Izabel Druzian ${ }^{\mathrm{I}}$
}

RESUMO

\begin{abstract}
O objetivo do trabalho foi desenvolver $e$ caracterizar uma embalagem biodegradável, utilizando como matriz polimérica a quitosana, plastificada com glicerol, bem como avaliar o efeito da adição de um aditivo antioxidante natural (urucum) nas embalagens na proteção antioxidante. As embalagens foram preparadas por casting contendo $1,5 \%$ de quitosana, $0,15 \%$ de glicerol e 0,25 a 1,0\% de urucum. O azeite de dende embalado com os filmes contendo o aditivo foi monitorado aos $0,7,15,30$ e 45 dias de armazenamento sob condições de oxidação acelerada $\left(63 \% U R / 30^{\circ} \mathrm{C}\right)$. $O$ azeite de dendê embalado no filme que continha o maior percentual de urucum $(1,0 \%)$ foi o que menos oxidou ao longo do período estudado. Constatouse que, à medida que aumentam as perdas de Fenóis Totais nas formulações dos filmes, ocorre uma redução nos aumentos do Índice de Peróxidos do produto embalado, demonstrando assim que, ao invés do produto, os compostos da embalagem é quem estão sofrendo oxidação.
\end{abstract}

Palavras-chave: embalagem ativa, fenóis, quitosana, urucum.

\section{ABSTRACT}

The objective was to develop and characterize a biodegradable packaging using chitosan as polymeric matrix, plasticized with glycerol, as well as evaluate the effect of adding a natural antioxidant additives (annatto) in antioxidant protection in packaging. The cans were prepared by casting containing $1.5 \%$ of chitosan, $0.15 \%$ glycerol and 0.25 to $1.0 \%$ of coloring. Palm oil packed with the films containing the additive was monitored at $0,7,15,30$ and 45 days of storage under accelerated oxidation conditions $\left(63 \% U R / 30^{\circ} \mathrm{C}\right)$. Palm oil packed in the film that contained the highest percentage of annatto (1.0\%) was the least oxidized during the study period. It was found that, as they increase the losses of phenolic compounds in the formulations of the films, there is a reduction in the peroxide increases the packaged product, thus demonstrating that instead of the product, the compounds of the package's who are suffering oxidation.

Key words: active packaging, phenols, chitosan, annatto.

\section{INTRODUÇÃO}

Geralmente, os materiais para a elaboração de embalagens têm sido selecionados com o objetivo de possuir a menor interação com o produto que acondicionam. Diante disso, as embalagens atuam como barreiras inertes, com a função principal de proteger o produto embalado, sem interagir com ele (VEIGA-SANTOS et al., 2005a). Entretanto, novas linhas de pesquisa têm surgido nas últimas décadas com o objetivo de desenvolver embalagens que interajam com o produto embalado, trazendo, portanto, uma vantagem adicional para estas. Muitas dessas embalagens ativas têm sido desenvolvidas a partir de matérias-primas naturais e renováveis, tanto a matriz polimérica como os aditivos funcionais incorporados, apresentando assim o caráter da biodegradabilidade. Alguns estudos revelam a incorporação de aditivos naturais nessas embalagens, como, por exemplo, extrato de ervamate (MACHADO, 2011), polpa de manga e acerola

IDepartamento de Análises Bromatológicas, Faculdade de Farmácia, Universidade Federal da Bahia (UFBA), Salvador, BA, Brasil.

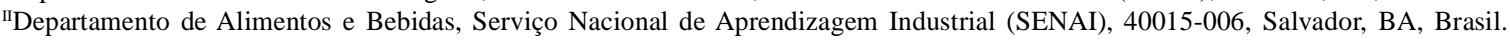
E-mail: brunam@fieb.org.br. Autor para correspondência.

IIIDepartamento de Ciência dos Alimentos, Escola de Nutrição, UFBA, Salvador, BA, Brasil. 
(SOUZA et al., 2011), dendê (GRISI et al., 2008), café e cacau (SILVA, 2009) entre outros, sendo assim planejadas para corrigir deficiências das embalagens passivas. Destaca-se ainda que essas embalagens ativamente funcionais contribuem para uma maior preservação do alimento, visto que os aditivos naturais incorporados auxiliam na manutenção das características sensoriais e microbiológicas destes alimentos, a depender da sua atividade funcional (VEIGA-SANTOS et al., 2005b; VEIGA \& DRUZIAN, 2007; MALI et al., 2010; MACHADO et al., 2010).

A quitosana é uma poliamina linear, caracterizada por sua origem renovável, biodegradável e pela sua capacidade de formar filmes resistentes, flexíveis, de difícil rompimento (JOHN \& THOMAS, 2008). Além disso, é um composto de grande interesse industrial por apresentar caráter bioativo, permeabilidade seletiva, ação polieletrônica, habilidade de quelação e capacidade adsortiva (THARANATHAN, 2003; SYNOWIECKI \& ALKHATEEB, 2003). Os filmes de quitosana apresentam, na condição seca, baixa permeação a gases, inferiores aos medidos para PET (Politereftalato de etileno) ou PVC (cloreto de polivinila) (ASSIS, 2010), sendo apropriados para embalar alimentos lipídicos. Outra possibilidade de aplicação em embalagens é através da incorporação de aditivos antioxidantes naturais, ampliando seu campo de utilização.

As reações de degradação por oxidação são consideradas como as mais importantes que ocorrem nos alimentos, já que são responsáveis pela deterioração dos produtos (FUKUMOTO \& MAZZA, 2000; LOULI et al., 2004; ALBU et al., 2004), limitando assim a conservação destes. Frequentemente, são adicionados, no produto ou na embalagem, substâncias antioxidantes, que são capazes de remover o oxigênio do meio ou impedir a reação em cadeia, produzida pelos radicais livres formados durante o processo de oxidação. Comumente, são utilizados antioxidantes sintéticos, dentre os quais, os mais utilizados são o BHA (Butil-hidroxianisol) e BHT (Butil-hidroxitolueno). Atualmente, a utilização dos antioxidantes sintéticos tem sido bastante questionada, devido ao aparecimento de diversos estudos que alegam que esses antioxidantes podem promover efeitos tóxicos e carcinogênicos no organismo. Devido a essa grande preocupação com a segurança alimentar da população, tem crescido a busca por produtos naturais que possam servir como fontes de antioxidantes, para substituir os antioxidantes sintéticos (MARTINEZ-TOME et al., 2001; BERNARDO-GIL et al., 2002; RIBEIRO et al., 2001). O urucum é considerado uma fonte natural rica em antioxidantes, como os compostos fenólicos (ALMEIDA et al., 1996; COSTA et al., 2008), chegando a apresentar até 1498,48 mg/g desses compostos em sua composição (LEMOS, 2008).

$\mathrm{O}$ objetivo deste trabalho foi avaliar a eficácia da incorporação de diferentes concentrações do urucum como aditivo antioxidante em filmes biodegradáveis a base de quitosana. A ação antioxidante dessas embalagens ativas foi realizada através do monitoramento das alterações químicas da embalagem e do produto embalado. Destacase que nenhum outro estudo foi encontrado na literatura utilizando urucum incorporado numa matriz polimérica de quitosana e agindo como uma embalagem ativa.

\section{MATERIAL E MÉTODOS}

Os filmes biodegradáveis foram preparados por casting, que consistiu no preparo de uma solução filmogênica, em que, inicialmente, dissolveu-se em ácido acético glacial 1\% (Vetec, Brasil) a quitosana (Sigma-Aldrich, Alemanha), o glicerol (Vetec, Brasil), utilizado como agente plastificante e o aditivo natural antioxidante, o urucum. A solução foi mantida sob agitação constante por $24 \mathrm{~h}$ para total dissolução do polissacarídeo (ASSIS \& SILVA, 2003). As formulações A (1,5\% de quitosana; $0,15 \%$ de glicerol; e $0,25 \%$ de urucum), B (1,5\% de quitosana; $0,15 \%$ de glicerol; $0,5 \%$ de urucum) e C (1,5\% de quitosana; $0,15 \%$ de glicerol; $1 \%$ de urucum) foram usadas para embalar azeite de dendê, com intuito de investigar o comportamento do aditivo antioxidante contido na embalagem durante armazenamento por 45 dias, em oxidação acelerada $\left(63 \%\right.$ UR, $\left.30^{\circ} \mathrm{C}\right)$. Como controle, foi utilizado o azeite de dendê embalado em filme de quitosana e plastificante sem adição de antioxidante (C1), embalado em PEBD (polietileno de baixa densidade) (C2) e sem embalagem, ou seja, exposto em placa de Petri (C3). As embalagens foram confeccionadas como sacos retangulares possuindo dimensões $5 \times 2 \mathrm{~cm}$ e, em cada uma, foram adicionados $8 \mathrm{~mL}$ de azeite de dendê. As embalagens foram seladas com uma seladora de bancada Sulpack, modelo SM 300 Light, de temperatura contínua.

A estabilidade oxidativa do azeite de dendê, em resposta à ação antioxidante das embalagens ativas foi monitorada por um período de 45 dias, através da determinação do índice de peróxido (IP). O IP do azeite de dendê embalado e exposto foi determinado pelo método titulométrico, de acordo com a AOAC 
Cd 8b-90 (2000), enquanto que a estabilidade dos antioxidantes nas embalagens ativas foi feita através do teor de Fenóis Totais (FT) por espectrofotometria (SWAIN \& HILLIS, 1959), sendo todas as análises realizadas em triplicata.

As medições de atividade de água (aw), permeabilidade ao vapor de água, umidade e sólidos totais das embalagens ativas pré-acondicionados $\left(52 \% \mathrm{UR}, 30^{\circ} \mathrm{C}\right)$ foram realizadas de acordo com a metodologia proposta por VEIGA-SANTOS et al. (2005a). A espessura das embalagens ativas foi avaliada através da espessura média, de 6 medições em posições aleatórias, por meio de micrômetro digital Mitutoyo. Todas as análises foram realizadas em triplicata. As propriedades mecânicas foram avaliadas a partir ds ensaios de tração, que foram realizados em uma máquina universal de ensaios (EMIC, modelo DL2000/700) com carga máxima de $20 \mathrm{KN}$, velocidade de $12,5 \mathrm{~mm} \mathrm{~min}^{-1}$ e temperatura de $25^{\circ} \mathrm{C}$, em seis corpos de prova para cada amostra (ASTM D-882, 2001).

Os resultados encontrados foram tratados pelo Teste de Tukey (ANOVA) através do programa Statistica 7.0 para identificar se as alterações nos parâmetros avaliados foram significativas ao nível de 95\% de significância.

\section{RESULTADOS E DISCUSSÃO}

$O$ efeito da incorporação de 0,25 , 0,5 e $1,0 \%$ do aditivo antioxidante natural nas embalagens $\mathrm{A}, \mathrm{B}$ e $\mathrm{C}$, respectivamente, durante o armazenamento, por um período de 45 dias, está apresentado na tabela 1 e indica que ocorrem perdas significativas nos FT após 45 dias de armazenamento. Entretanto, é possível constatar que, mesmo após o período avaliado, os compostos fenólicos do aditivo incorporado permanecem viáveis nas embalagens. $\mathrm{O}$ mesmo produto (azeite de dendê) foi embalado por SOUZA et al. (2011) em filmes de amido de mandioca incorporados com aditivos naturais antioxidantes (polpa de manga e acerola). Também foi verificado pelos autores que, após 45 dias de armazenamento, as formulações de filmes apresentaram perdas nos FT, variando de 17,80 a $36,12 \%$. MACHADO (2011) avaliou a ação antioxidante de filmes de amido de mandioca contendo nanocelulose como reforço mecânico e extrato de erva-mate como aditivo antioxidante, ao embalar azeite de dendê. Foi relatado que houve uma diminuição no valor de FT dos filmes de 102,70 para $60,72 \mathrm{mg} \mathrm{g}^{-1}$ após 40 dias de armazenamento.

Através do monitoramento do IP do azeite de dendê embalado nas diferentes formulações, foi observado um aumento no teor inicial desse parâmetro para o produto embalado após o período estudado (45 dias), para as três embalagens ativas (A, B e C) e nos controles (C1, C2 e C3) (Tabela 2 e Figura 1). Entretanto, é importante destacar que o azeite de dendê embalado nos filmes ativos A, B e C apresentou um comportamento distinto ao longo dos 45 dias de armazenamento, com diferenças significativas entre as amostras $(\mathrm{P}>0,05)$, já que estas apresentavam diferentes percentuais de aditivo incorporados. A partir dos resultados apresentados na tabela 2 e figura 1, é possível observar que houve um efeito protetor mais amplo apresentado pela embalagem $\mathrm{C}$, quando comparado com as embalagens A e B, e isso é justificado pelo maior percentual de aditivo antioxidante (urucum) presente nessa formulação, demonstrando, assim, que, quanto maior o percentual de aditivo na embalagem, menor é o processo de oxidação sofrido pelo azeite de dendê. Resultados semelhantes ao deste estudo foram encontrados por GRISI et al. (2008), quando avaliaram o processo de oxidação do óleo de soja embalado com filmes de

Tabela 1 - Teores de fenóis totais $(\mathrm{mg} / \mathrm{g})$ apresentados pelas embalagens durante 45 dias de armazenamento.

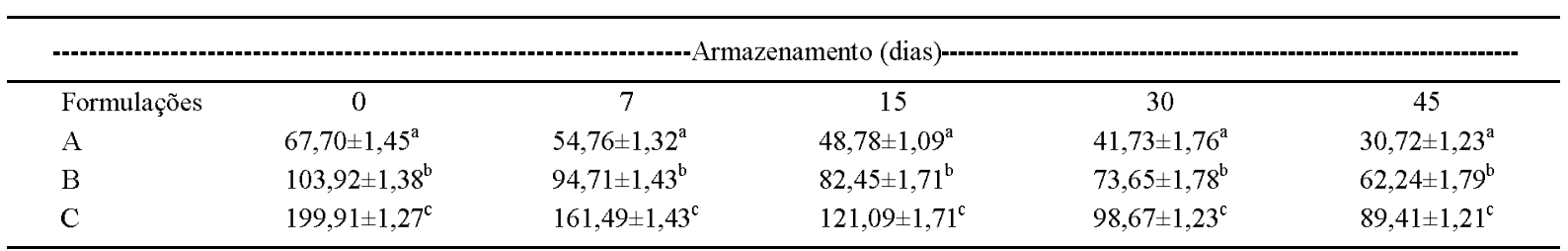

Valores que apresentam a mesma letra, numa mesma coluna, não apresentam diferenças significativas $(\mathrm{P}>0,05)$ pelo Teste de Tukey a $95 \%$ de confiança.

A ( $1,5 \%$ de quitosana; $0,15 \%$ de glicerol; e $0,25 \%$ de urucum); B (1,5\% de quitosana; $0,15 \%$ de glicerol; $0,5 \%$ de urucum); e C (1,5\% de quitosana; $0,15 \%$ de glicerol; $1 \%$ de urucum);

Ciência Rural, v.43, n.3, mar, 2013. 
Tabela 2 - Determinação dos teores de índice de peróxidos $\left(\mathrm{meq} \mathrm{kg}^{-1}\right)$ apresentados pelo azeite de dendê nos dias $0,7,15,30$ e 45 e percentual de aumento durante armazenamento nas embalagens ativas e nos controles.

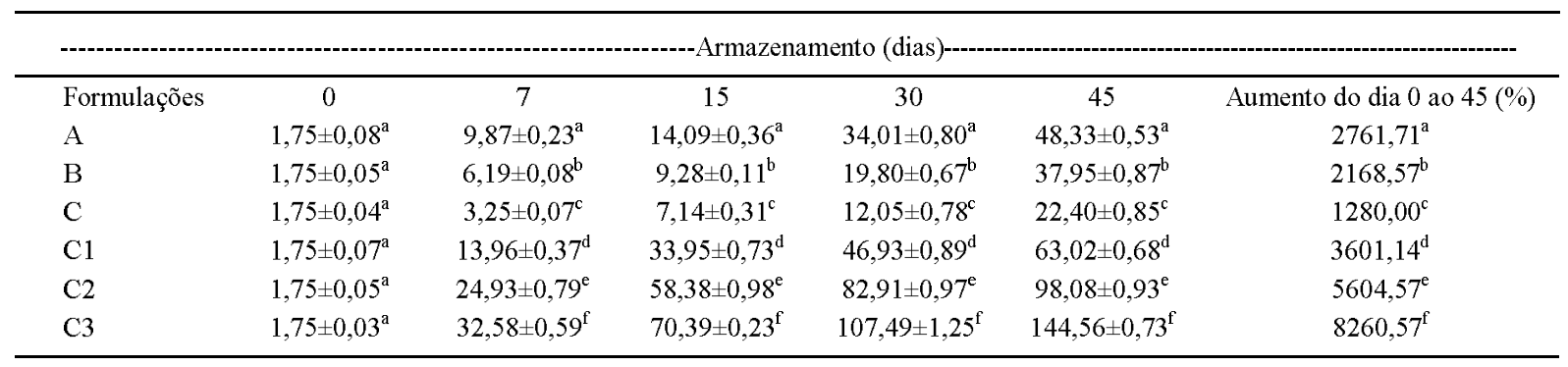

Formulações A (1,5\% de quitosana; $0,15 \%$ de glicerol; e $0,25 \%$ de urucum), B (1,5\% de quitosana; $0,15 \%$ de glicerol; $0,5 \%$ de urucum) e C (1,5\% de quitosana; $0,15 \%$ de glicerol; $1 \%$ de urucum); $\mathrm{C} 1: 1,5 \%$ de quitosana e $0,15 \%$ de glicerol; C2: embalagem de PEBD; C3: sem embalagem.

Valores que apresentam a mesma letra, numa mesma coluna, não apresentam diferenças significativas $(\mathrm{P}>0,05)$ pelo Teste de Tukey a $95 \%$ de confiança.

amido contendo o extrato do fruto do dendê como aditivo antioxidante. Foi relatado pelos autores que, quanto maior o percentual de aditivo presente na embalagem, menor era o IP apresentado pelo óleo de soja. Através de um planejamento de superfície de resposta, MACHADO (2011) e REIS (2011) constataram que, quanto maior o teor de extrato de erva-mate, utilizado como aditivo antioxidante, em embalagens de amido de mandioca, menor era a oxidação apresentada pelo produto embalado (azeite de dendê).

$\mathrm{O}$ azeite de dendê embalado em todos os filmes ativos (A, B e C) apresentou um menor aumento no IP $(\mathrm{P}<0,05)$, quando comparados aos apresentados pelo produto embalado com os três controles, indicando a eficácia do extrato de urucum como aditivo antioxidante. Os resultados encontrados neste trabalho também mostram que, mesmo sem o aditivo antioxidante, a embalagem de quitosana (C1) têm um efeito protetor maior da oxidação do azeite $(\mathrm{P}<0,05)$, quando comparado à embalagem de PEBD (C2). Esses resultados mostram que os filmes de quitosana podem representar uma barreira mais eficaz ao oxigenio do que os filmes de PEBD. Além disso, essa matriz apresenta propriedades antioxidantes já relatadas em outros estudos (ASSIS \& SILVA, 2003; COSTA-JR,

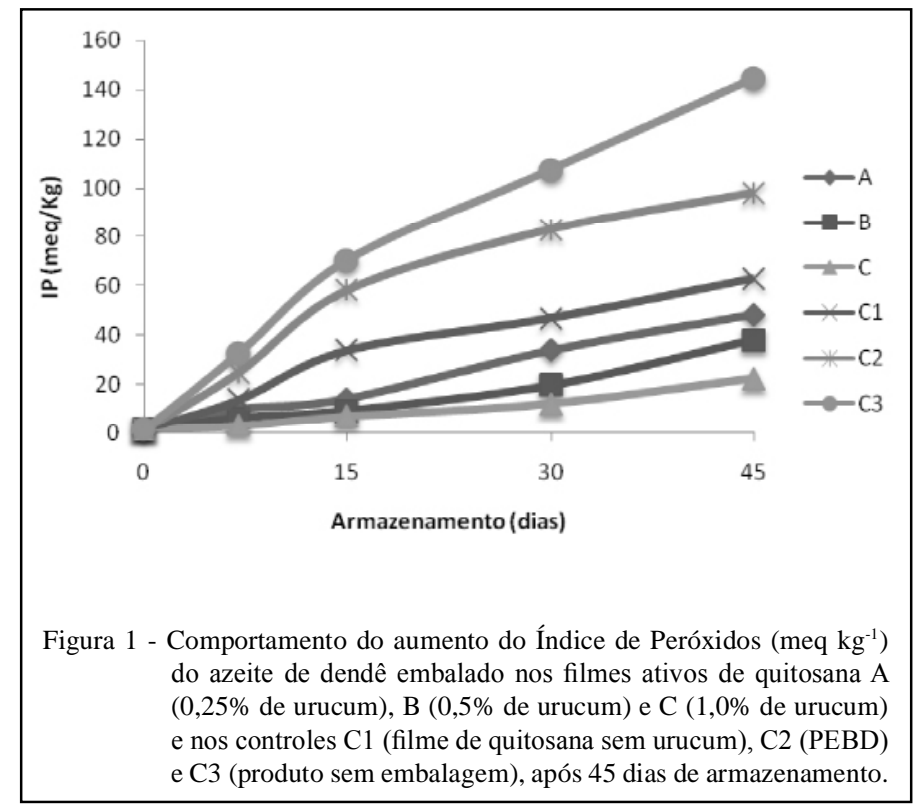

Ciência Rural, v.43, n.3, mar, 2013. 
2008; ASSIS, 2010). Portanto, o efeito antioxidante protetor está relacionado com a presença do aditivo incorporado e também da matriz polimérica.

Como esperado, dentre os controles avaliados, o azeite exposto (C3), ou seja, sem nenhuma barreira de proteção, apresentou o maior aumento do IP ao longo de 45 dias. Os resultados obtidos neste estudo sobre a ação antioxidante de aditivos naturais, quando incorporados em filmes contendo uma matriz polimérica biodegradável, pode ser confirmado através de outros trabalhos encontrados na literatura (GRISI et al., 2008; JUNQUEIRA et al., 2009; SOUZA et al., 2011; MACHADO, 2011; MACHADO et al., 2012).

Para avaliar se a incorporação do urucum exerce alguma influência nas propriedades mecânicas e de barreira das embalagens formuladas a partir de uma matriz polimérica de quitosana, foram realizados os ensaios de tração e as determinações das propriedades de barreira (Tabela 3). O controle C1 (sem a incorporação do urucum) foi utilizado para comparar os resultados obtidos. A partir dos valores encontrados para umidade e atividade de água, as embalagens elaboradas neste estudo são consideradas produtos de umidade intermediária. Em relação às propriedades mecânicas, foram encontrados ótimos valores para o módulo de Young, tensão máxima e deformação, sendo essas embalagens consideradas como embalagens poliméricas biodegradáveis resistentes. Destaca-se que os valores de Módulo de Young e tensão máxima encontrados neste estudo são semelhantes aos relatados por SILVA et al. (2012) e MACHADO (2011), que incorporaram nanocelulose da fibra de piaçava e da fibra de coco, respectivamente, para reforçar mecanicamente as embalagens de amido de mandioca. Constata-se, portanto, que as embalagens de quitosana apresentam-se mais resistentes do que as embalagens biodegradáveis de amido de mandioca, já que não necessitam de nenhum aditivo de reforço.

A partir dos resultados apresentados na tabela 3 , observa-se que a incorporação do aditivo antioxidante natural (urucum) na matriz polimérica de quitosana não alterou significativamente $(\mathrm{P}>0,05)$ as propriedades de barreira das embalagens biodegradáveis. Comportamento semelhante foi observado para as propriedades mecânicas, isto é, não houve alteração significativa no módulo de Young, tensão máxima e percentual de deformação das embalagens. Com isso, pode-se afirmar que as embalagens passaram a ter uma vantagem adicional de fornecer a propriedade ativa, ou seja, uma propriedade antioxidante, sem apresentar alterações nas propriedades de barreira e mecânicas.

\section{CONCLUSÃO}

A partir dos resultados obtidos, é possível concluir que o desenvolvimento de embalagens ativas, utilizando o urucum como fonte de compostos antioxidantes incorporados à matriz de quitosana é viável. Essa embalagem pode ser aplicada para embalar alimentos lipídicos, como óleos e gorduras, para evitar a oxidação desses produtos. Vale ressaltar ainda que a incorporação desse aditivo natural não altera as propriedades mecânicas e de barreira das embalagens, permitindo assim o seu desenvolvimento e comercialização como uma embalagem ativa antioxidante.

Tabela 3 - Médias das análises de caracterização das embalagens ativas (A, B e C) e do controle (C1). Ep (espessura - mm); aw (atividade de água); ST (sólidos totais - \%); Umidade (\%); PVA (permeabilidade ao vapor de água - $\mathrm{gH}_{2} \mathrm{O} . \mu \mathrm{m} \mathrm{m} \mathrm{m}^{-2}$.h.mmHg); $\mathrm{E}$ ( Módulo de Yonng - MPa); $\sigma$ (Tens̃̃o Máxima - MPa);c (Deformação - \%).

\begin{tabular}{|c|c|c|c|c|c|c|c|c|}
\hline $\mathrm{F}$ & Ep & aw & ST & $\mathrm{U}$ & PVA & $\mathrm{E}(\mathrm{MPa})$ & $\sigma(\mathrm{MPa})$ & $\varepsilon(\%)$ \\
\hline $\mathrm{A}$ & $0,085 \pm 0,02^{\mathrm{a}}$ & $0,589 \pm 0,15^{\mathrm{a}}$ & $78,94 \pm 0,32^{\mathrm{a}}$ & $21,06 \pm 0,21^{\mathrm{a}}$ & $2,58 \times 10^{-6} \pm 0,03^{\mathrm{a}}$ & $212,24 \pm 1,21^{\mathrm{a}}$ & $13,97 \pm 1,32^{\mathrm{a}}$ & $23,90 \pm 1,07^{\mathrm{a}}$ \\
\hline $\mathrm{B}$ & $0,082+0,01^{\mathrm{a}}$ & $0,584+0,14^{\mathrm{a}}$ & $79,53+0,01^{\mathrm{a}}$ & $20,47 \pm 0,01^{\mathrm{a}}$ & $2,57 \times 10^{-6} \pm 0,06^{\mathrm{a}}$ & $209,83+0,24^{\mathrm{a}}$ & $14,13+1,12^{\mathrm{a}}$ & $23,20+1,10^{\mathrm{a}}$ \\
\hline $\mathrm{C}$ & $0,084+0,01^{\mathrm{a}}$ & $0,588-0,11^{\mathrm{a}}$ & $79,81+0,32^{\mathrm{a}}$ & $20,19+0,34^{\mathrm{a}}$ & $2,53 \times 10^{-6} \pm 0,05^{\mathrm{a}}$ & $211,87+0,76^{\mathrm{a}}$ & $14,98+1,20^{\mathrm{a}}$ & $23,76+1,34^{\mathrm{a}}$ \\
\hline $\mathrm{C} 1$ & $0,089 \pm 0,01^{\mathrm{a}}$ & $0,581+0,16^{\mathrm{a}}$ & $79,24+0,19^{\mathrm{a}}$ & $20,76-0,56^{\mathrm{a}}$ & $2,59 \times 10^{-6}+0,02^{\mathrm{a}}$ & $210,83+1,56^{\mathrm{a}}$ & $14,34-1,76^{\mathrm{a}}$ & $23,98+1,21^{\mathrm{a}}$ \\
\hline
\end{tabular}

Valores que apresentam a mesma letra, numa mesma coluna, não apresentam diferenças significativas $(\mathrm{P}>0,05)$ pelo Teste de Tukey a $95 \%$ de confiança.

Formulações $A(1,5 \%$ de quitosana; $0,15 \%$ de glicerol; e $0,25 \%$ de urucum), B (1,5\% de quitosana; $0,15 \%$ de glicerol; $0,5 \%$ de urucum) e $C$ ( $1,5 \%$ de quitosana; $0,15 \%$ de glicerol; $1 \%$ de urucum); $\mathrm{C} 1: 1,5 \%$ de quitosana e $0,15 \%$ de glicerol; C2: embalagem de PEBD; C3: sem embalagem.

Ciência Rural, v.43, n.3, mar, 2013. 


\section{REFERÊNCIAS}

ALBU, S. et al. Potential for the use of ultrasound in the extraction of antioxidants from Rosmarinus officinalis for the food and pharmaceutical industry. Ultrasonics Sonochemistry, v.11, p.261-265, 2004. Disponível em: <http://dx.doi.org/10.1016/j. ultsonch.2004.01.015>. Acesso em: 12 jan. 2012. doi: 10.1016/j. ultsonch.2004.01.015.

ALMEIDA, J.L. et al. Indução de brotações em explantes de segmentos de folhas de plântulas de urucueiro em diferentes citocininas. Ciência Rural, v.26, n.1, p.45-49, 1996. Disponível em: <http://dx.doi.org/10.1590/S0103-84781996000100009>. Acesso em: 12 jan. 2012. doi: 10.1590/S0103-84781996000100009.

AOAC (ASSOCIATION OF OFFICIAL ANALYTICAL CHEMISTS). Official methods of analysis Cd $8 b-90$. Washington, 2000. 1141p.

ASSIS, O.B.G.; SILVA, V. Caracterização estrutural e da capacidade de absorção de água em filmes finos de quitosana processados em diversas concentrações. Polímeros, v.13, n.4, p.223-228, 2003. Disponível em: <http://www.revistapolimeros. org.br/v13n4/3>. Acesso em: 22 jan. 2012. doi: 10.1590/S010414282003000400006

ASSIS, O.B.G. Alteração do caráter hidrofílico de filmes de quitosana por tratamento de plasma de HMDS. Química Nova, v.33, n.3, p.603-606, 2010. Disponível em: <http://dx.doi. org/10.1590/S0100-40422010000300022>. Acesso em: 20 jan. 2012. doi: 10.1590/S0100-40422010000300022.

ASTM (AMERICAN SOCIETY FOR TESTING AND MATERIALS). Standard test method for tensile properties of thin plastic sheeting ASTM D882-00. Philadelphia, 2001. 9p.

BERNARDO-GIL, M.G. et al. Produção de extractos para a indústria alimentar: uso de fluidos supercríticos. Boletim de Biotecnologia, v.73, p.14-21, 2002.

COSTA, A.J.C. et al. Abelhas (Hymenoptera: Apoidea) visitantes das flores de urucum em Vitória da Conquista, BA. Ciência Rural, v.38, n.2, p.534-537, 2008. Disponível em: <http://dx.doi. org/10.1590/S0103-84782008000200039>. Acesso em: 20 jan. 2012. doi: 10.1590/S0103-84782008000200039.

COSTA JR. et al. Preparação e caracterização de blendas de quitosana/poli(álcool vinílico) reticuladas quimicamente com glutaraldeído para aplicação em engenharia de tecido. Química Nova, v.31, n.6, p.1460-1466, 2008. Disponível em: <http://dx.doi. org/10.1590/S0100-40422008000600034>. Acesso em: 17 fev. 2012. doi: 10.1590/S0100-40422008000600034.

FUKUMOTO, L.R.; MAZZA, G. Assessing antioxidant and prooxidant activities of phenolic compounds. Journal of Agricultural and Food Chemistry, v.48, n.8, p.3597-3604, 2000. Disponível em: 〈http://pubs.acs.org/doi/abs/10.1021/jf000220w〉. Acesso em: 19 fev. 2012. doi: 10.1021/jf000220w.

GRISI, C.V.B. et al. Evaluation of the viability of incorporating natural antioxidants in bio-based packagings. Nova Science Publishers - Food Chemistry Research Developments, v.1, p.111,2008

JUNQUEIRA, M.S. Efeito de embalagens ativas no escurecimento enzimático de batatas (Solanum tuberosum) fatiadas e minimamente processadas. Ciências Agrárias, v.30, n.3, p.613618, 2009. Disponível em: <http://www.uel.br/revistas/uel/index. php/semagrarias/article/view/3569>. Acesso em: 01 fev. 2012.

LEMOS, A.R. Caracterização físico-química, bioquímica e avaliação da atividade antioxidante em genótipos de urucueiros (Bixa Orellana L.). 2008. 65f. Dissertação (Mestrado em Engenharia de Alimentos) - Faculdade de Engenharia de Processos de Alimentos, Universidade Estadual do Sudoeste da Bahia, BA.

LOULI, V. et al. Recovery of phenolic antioxidants from wine industry by-products. Bioresource Technology, v.92, p.201-208, 2004. Disponível em: <http://dx.doi.org/10.1016/j. biortech.2003.06.002>. Acesso em: 07 mar. 2012. doi: 10.1016/j. biortech.2003.06.002.

MACHADO, B.A.S. et al. Processo para a preparação de nanobiocompósitos tipo filmes flexíveis reforçados com nanopartículas de celulose tipo nanowhiskers. Patente protocolizada no INPI, 2010.

MACHADO, B.A.S. Desenvolvimento e caracterização de filmes flexíveis de amido de mandioca com nanocelulose de coco. 2011. 161f. Dissertação (Mestrado em Ciências de Alimentos) Faculdade de Farmácia, Universidade Federal da Bahia, BA.

MACHADO, B.A.S et al. Desenvolvimento e avaliação da eficácia de filmes biodegradáveis de amido de mandioca com nanocelulose como reforço e com extrato de erva-mate como aditivo antioxidante. Ciência Rural, v.42, n.11, p. 2085-2091, 2012. Disponível em: <http://dx.doi.org/10.1590/S0103-84782012001100028>. Acesso em: 14 out. 2012. doi: 10.1590/S0103-84782012001100028.

MALI, S. et al. Filmes de amido: produção, propriedades e potencial de utilização. Ciências Agrárias, v.31, n.1, p.137-156, 2010. Disponível em: <http://www.uel.br/revistas/uel/index.php/ semagrarias/article/view>. Acesso em: 30 jan. 2012.

MARTINEZ-TOME, $M$. et al. Antioxidant properties of Mediterranean spices compared with common food additives. Journal of Food Protection, v.64, n.9, p.14121419, 2001. Disponível em: <http://www.ncbi.nlm.nih.gov/ pubmed/11563520>. Acesso em: 30 jan. 2012.

REIS, L.C.B. Formulação e caracterização de filmes biodegradáveis de fécula de mandioca e incorporados com polpa de manga e extrato de erva-mate, e seu efeito na preservação de alimentos. 2011. 151f. Dissertação (Mestrado em Ciências de Alimentos) - Faculdade de Farmácia, Universidade Federal da Bahia, BA.

RIBEIRO, M.A. et al. Melissa officinalis L.: study of antioxidant activity in supercritical residues. Journal of Supercritical Fluids, v.21, n.1, p.51-60, 2001. Disponível em: <http://dx.doi. org/10.1016/S0896-8446(01)00078-X>. Acesso em: 25 jan. 2012. doi: 10.1016/S0896-8446(01)00078-X.

SILVA, L.T. Desenvolvimento e avaliação de embalagens biodegradáveis com ação antioxidante contendo café e cacau. 2009. 148f. Dissertação (Mestrado em Ciência de Alimento) Faculdade de Farmácia, Universidade Federal da Bahia, BA.

SILVA, J.B. etal. Cassava starch-based films plasticized with sucrose and inverted sugar and reinforced with cellulose nanocrystals. Journal of Food Science, v.77, p.14-19, 2012. Disponível em: <http://www.ncbi.nlm.nih.gov/pubmed/22582979>. Acesso em: 21 fev. 2012. 
SOUZA, C.O. et al. Mango and Acerola pulps as antioxidant additives in Cassava starch bio-based film. Journal of Agricultural and Food Chemistry, v.59, n.6, p.2248-2254, 2011. Disponível em: <http://pubs.acs.org/doi/abs/10.1021/jf1040405>. Acesso em: 02 jan. 2012. doi: 10.1021/jf104040.

SWAIN, T.; HILLIS, W.E. The phenolic constituents of Prunus doméstica: the quantitative analysis of phenolic constituents. Journal of the Science of Food and Agriculture, v.10, p.6368, 1959. Disponível em: <http://pubs.acs.org/doi/abs/10.1021/ la020475z>. Acesso em: 24 jan. 2012. doi: 10.1021/la020475z.

VEIGA-SANTOS, P. et al. Mechanical properties, hydrophilicity and water activity of starch-gum films: Effect of additives and deacetylated xanthan gum. Food Hydrocolloids, v.19, p.341-349, 2005a. Disponível em: <http://dx.doi.org/10.1016/j. foodhyd.2004.07.006>. Acesso em: 11 fev. 2012. doi: 10.1016/j. foodhyd.2004.07.006

VEIGA-SANTOS, P. et al. Microstructure and color of starch-gum films: Effect of additives and deacetylated xanthan gum. Part 2. Food Hydrocolloids, v.19, n.6, p.1064-1073, 2005b. Disponível em: <http://dx.doi.org/10.1016/j.foodhyd.2005.02.007>. Acesso em: 11 fev. 2012. doi: 10.1016/j.foodhyd.2005.02.007.

VEIGA-SANTOS, P.; SCAMPARINI, A.R.P. Indicador irreversível de temperatura utilizando carboidratos. PI0403610-7 A2, patente protocolizada no INPI, 2004.

VEIGA, P.S.; DRUZIAN, J.I. Absorvedores de umidade/ antiembaçantes biodegradáveis a base de amido/fécula para serem aplicados em produtos de higiene pessoal, alimentícios ou farmacêuticos. Patente protocolizada no INPI, 2007. 